\title{
Dynamic Minkowski Sums Under Scaling
}

\author{
Evan Behar and Jyh-Ming Lien \\ MASC group, Dept. of Computer Science, George Mason University
}

\begin{abstract}
In many common real-world and virtual environments, there are a significant number of repeated objects, primarily varying in size. Similarly, in many complex machines, there are a significant number of parts which also vary in size rather than shape. This repetition saves in both design and production costs. Recent research in robotics has also shown that exploiting workspace repetition can significantly increase efficiency. In this paper, we address the need to support computation reuse in fundamental operations. To this end, we propose an algorithm to reuse the computation of the Minkowski sum when an object is transformed by uniform scaling. The Minkowski sum is a fundamental operation in many areas of robotics, such as motion planning, computer vision, and mathematical morphology, and has been studied extensively over the last four decades. We present two methods for dynamically updating Minkowski sums under scaling, the first of which updates the sum under uniform scaling of arbitrary polygons and polyhedra, and the second of which updates the sum under non-uniform scaling of convex polyhedra. Ours are the first methods that study the M-sum under this type of transformation. Our results show speed gains between one and two orders of magnitude over recomputing the Minkowski sum from scratch for each repeated object, and we discuss applications for motion planning, CAD, rapid prototyping, and shape decomposition.
\end{abstract}

Key words: Minkowski sum, Kinematic geometry, Geometry processing

\section{Introduction}

If you examine your surroundings, it should not be difficult to spot objects that have similar or even identical shapes. If you are in a study room, most books, file folders, and stationery have this property. If you are in a kitchen, most likely you will find many similar cups, dishes, bottles, cans and utensils.

Many such similar objects can also be found in other indoor environments such as offices, classrooms, factories, airports, and hospitals. In outdoor environments one finds cars, bicycles, benches, lamp posts, and traffic signs all have similar shapes. If we design a robot to interact with, navigate in, and manipulate these environments, taking the repetition into consideration and reusing the computation for similar objects will make these tasks easier and faster.

Furthermore, we can also see that almost every complex object, ranging from a washing machine, to a car engine, to a space shuttle, is made of many components with similar shapes. During the design and prototyping stage, the shape of these components may also have to go through multiple revisions.

Thus, if a virtual prototyping system has the ability to recognize the similarity between these components as well as between various design revisions, then tasks such as assembly/disassembly planning and part removal can be performed much more efficiently. Object reuse is also com- mon in virtual environments, video games and 3D graphics. Similar objects with different scales are usually reused in different parts of a scene graph, different shots in an animation and different areas and levels in a video game in order to save both rendering and design costs. If similar objects can also share computations, such as collision detection and response, then operations such as haptic rendering and physically-based simulation can be dramatically accelerated.

In all of the aforementioned examples, the Minkowski sum plays an important and fundamental role. It is an important and basic operation for tasks such as configuration space mapping, penetration depth estimation, and collision detection. The Minkowski sum of two shapes, $P$ and $Q$ is defined as $P \oplus Q=\{p+q \mid p \in P, q \in Q\}$. Though its study dates back to the early 70 s (see the cited surveys for more details $[15,29,14])$, recent work has also emerged studying the idea of dynamic Minkowski sums, rapid methods of updating the Minkowski sum under various transformations such as rotation $[6,7,26]$. However, as we have seen in our earlier discussion, rotation is not the only common transformation under which the Minkowski sum may be recomputed.

In this paper, we examine the case of a common and basic deformation: scaling. Scaling of an object $P$ by a scale factor $s$ is simply the transformation of each vertex $v=\left(v_{1}, v_{2}, \ldots, v_{d}\right)$ in $P$ to a new position $v^{\prime}=$ 


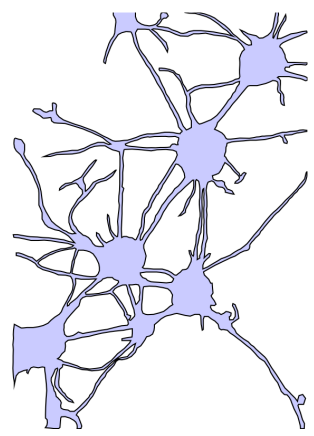

(a) input

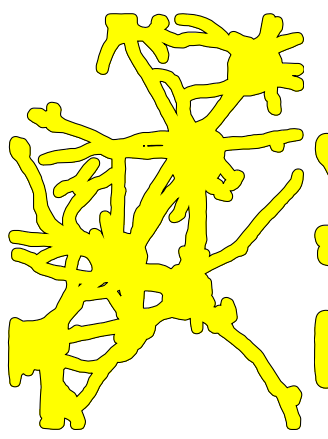

(b) $s=1$

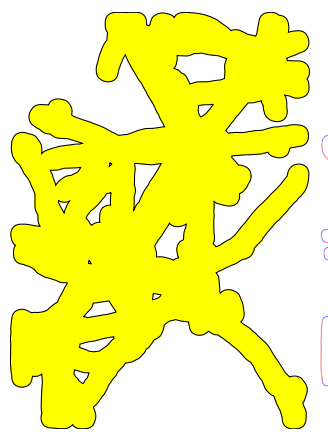

(c) $s=2$

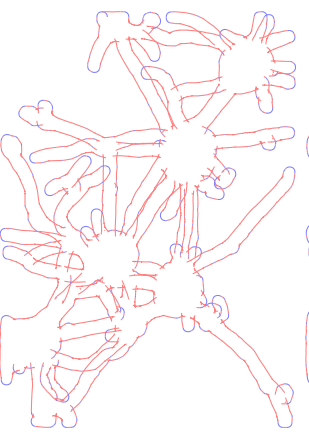

(d) convolution $s=1$

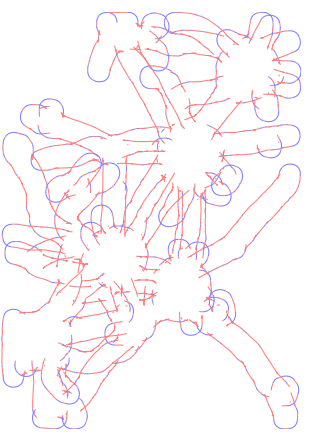

(e) convolution $s=2$

Fig. 1. Comparison of the Minkowski sums [(b) and (c)], and the reduced convolutions [(d) and (e)] for neuron \& disc at scales $s=1$ and $s=2$. The neuron polygon shown in (a) has 18 holes and 1,815 vertices and the disc is represented as a polygon with 32 vertices. The topology of the Minkowski sum changes between scales. The definition of the reduced convolution is in Section 3.

$\left(s_{1} v_{1}, s_{2} v_{2}, \ldots, s_{d} v_{d}\right)$, where for $v \in \mathbb{R}^{d}$ and $s_{i} \in(0, \infty)$. Uniform scaling consists of the special case where $s_{1}=$ $s_{2}=\ldots=s_{d}$.

Our goal is to develop an algorithm that can efficiently update the Minkowski sum when the objects are scaled without recomputing the Minkowski sum from scratch. The main challenge comes from the fact that the Minkowski sum can change drastically when the underlying objects are scaled. For example, in Fig. 1, when the disc is scaled to twice its original size, not only the geometry but also the topology of the Minkowski sum changes.

Main Contribution. Our main contributions in this paper are two exact, output-sensitive algorithms whose computation time depends on the amount of scale and therefore depends on the number of changes to the Minkowski sum due to scaling.

We base our first method on the Minkowski sum methods in our previous work $[8,23]$, which computes a reduced convolution of the two polygons or two polyhedra (see definition in Section 3). In 2D, the reduced convolution is a subset of the full convolution which omits contributions from the reflex vertices of the input polygons. Reflex vertices are vertices whose interior angles are greater than 180 degrees. Two reduced convolutions are illustrated in Fig. 1.

Given this reduced convolution, denoted by $P \widehat{\otimes} Q$, we show that there is an efficient way to update both $2 \mathrm{D}$ Minkowski sums under scaling regardless of the convexity of the input models (Section 4). The set of intersecting faces in the Minkowski sum changes only at a finite set of scale values. We call these values critical events, and it is on this notion that our idea rests. As shown in our experiments (Section 7), this method is generally one to two orders of magnitude faster than recomputing the Minkowski sum from scratch.

We show that this method can be extended naturally to 3D (in Section 5). However, we also show that it is not practical to do so due to time and space complexity constraints as the convolution in $3 \mathrm{D}$ is much more complex. To address this, in Section 6, we introduce the second method, which dynamically identifies compatibility errors in the convolution for convex polyhedra and corrects these errors without precomputing when they will occur. We show that this method supports non-uniform scaling, provides significant speed improvements over brute force, and does not en- counter the complexity constraints of precomputation.

In addition to reusing computations for the obvious purpose of increasing the computation efficiency, in Section 8 we also demonstrate that our method can be extended to answer the query, "Given the specification of manufacturing tolerance, what are the largest and smallest scales of $P$ for which a given path is valid?" This query is useful in rapid prototyping, for determining the necessary scale for parts to fit into an assembly. With this information we can check whether there exists a scale for a part $P$ that can guarantee it fits into the assembly properly. The proposed method also finds unusual applications in shape decomposition, where it can be used to identify structural features based on multi-scale convolutions.

To the best of our knowledge, this paper presents the first work which considers dynamic Minkowski sums under scale and our results are encouraging. However, there are two main limitations for the first method. First, it is only able to handle uniform scaling, as it relies on the idea that the facet normals do not change under scaling. Secondly, it is impractical in more than two dimensions due to its large time and space complexities. In three or more dimensions, we quickly run up against the curse of dimensionality. The main limitation of the second method is its inability to handle non-convex inputs, since it relies on the principle that each facet in the convolution will be associated with at most one vertex in the input models.

\section{Related work}

For methods computing static Minkowski sum, detailed surveys can be found in $[15,29,14,5]$. A brief discussion of some recent works can also be found in Section 3 .

Currently there exist few works in computing Minkowski sums for geometric objects under rotation. Many early works can be found in configuration-space (C-space) mapping [24,31,20,28]. These methods generate slices of $\mathrm{C}$-space obstacles (C-obst) at fixed rotational resolution using the Minkowski sums and ignore the temporal and spatial coherence between slices. To avoid this problem, the methods proposed by Avnaim et al. [2] and Brost [9] focus on the idea of contact surfaces, which have a close relationship to convolution (defined in Section 3). For nonconvex polygons, part of the contact region may belong to 
the interior of the C-obst. Therefore, all contact regions are tested for intersections and are trimmed around the boundary created by the intersections. Even for simple shapes many contact regions are trimmed. As a result, significant computation is wasted. In our recent work [6], we address this issue (1) by reducing the number of contact regions and (2) by explicitly determining the values of rotation $\theta$ for which the structure of the Minkowski sum changes. Our method then computes C-obst by smoothly sweeping the segments of the Minkowski sums between consecutive critical rotations.

However, these methods are limited to $2 \mathrm{D}$ polygons and attempts to extend contact surfaces to higher dimensional space are still strictly theoretical. [10]. Recently, Mayer et al. [26] have studied transformation of the Minkowski sum under rotation using the idea of a criticality map, which stores the rotation values at which the combinatorial structure of the Minkowski sum changes.

Obtaining the full representation of the combinatorial structure the Minkowski sums of 3D polyhedra under rotation is expensive. In many cases, such as penetration depth estimation, it is also unnecessary. In our recent work [22,7], we observe that significant performance gains can be obtained if the Minkowski sum is dynamically updated by identifying and correcting the errors under the given rotation. When the input polyhedra are convex, we use the observation that there are only $O(n)$ of certain types of facets in the sum, where $n$ is the size of input polyhedra, and that all of the remaining erroneous facets can be identified by first locating a linear subset of facets and then performing gradient descent.

\section{Preliminaries}

In order to provide sufficient background for the readers, we briefly discuss the suitability of existing static Minkowski sum methods for dynamic Minkowski sums under scaling.

Many earlier methods based on convex decomposition compute the pairwise Minkowski sums of the components, and then merge all of the pairwise Minkowski sums. Although the main idea of this approach (first proposed by Lozano-Pérez [24]) is simple, the decomposition and the union steps can be very tricky and have become the main focus of several recent works $[1,13,18,29,21]$.

An additional trouble for the application at hand is that when $P$ is scaled, updating the Minkowski sum using a convex decomposition means that once all pairwise Minkowski sums are updated, their union must then be recomputed. This presents a significant hurdle to creating dynamic Minkowski sums using the convex decomposition method.

Due to this, convolution-based approaches becomes more appealing. The convolution of two shapes $P$ and $Q$, denoted as $P \otimes Q$, is a structure produced by combining primitives from the two shapes $[16,17]$. It is known that the convolution is a superset of the Minkowski sum, that is, $P \oplus Q \subseteq$ $P \otimes Q$. More specifically, it is known that $\partial(P \oplus Q)=$ $\partial(P \otimes Q)$.

The main challenge of the convolution-based methods is in trimming edges and facets in the interior of

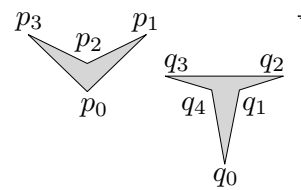

(a) $P$ (left), $Q$ (right)

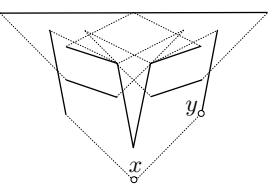

(b) $P \otimes Q$

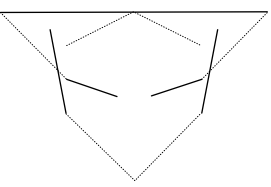

(c) $P \widehat{\otimes} Q$
Fig. 2. Polygons $P$ and $Q$, their convolution $P \otimes Q$ and reduced convolution $P \widehat{\otimes} Q$. In the figure showing $P \otimes Q, x=p_{0}+q_{0}$ and $y=p_{1}+q_{0}$. Edge $\overline{p_{0} p_{1}}$ is compatible with $q_{0}$, because $\overline{p_{0} p_{1}}$ is on the counterclockwise turn between $\overline{q_{4} q_{0}}$ and $\overline{q_{0} q_{1}}$.

the convolution to obtain the Minkowski sum boundary $[15,19,30,3,4,27]$. For example, Wein [30] computes the arrangement induced by the convolution and keeps the cells with non-zero winding numbers. The arrangement is a data structure representing the subdivision of the space created by the convolution [11]. The winding number of the convolution of a point $p$ is the number of times that convolution travels counterclockwise around $p$.

As it will become clear later, convolution methods are more suitable for dynamically updating Minkowski sums because the combinatorial structure of the convolution typically remains the same when the change to the input model is small. Additionally, the relationship between the convolution edges can be updated in a straightforward way when the input models are transformed smoothly.

In the case of uniform scaling, the structure of the convolution never changes and the structure of the arrangement only changes at fixed scale factors, so-called critical scales. This property enables intermediate forms to be updated rapidly. Additionally, there are far fewer primitives in the convolution of two shapes than there are pairwise Minkowski sums from their decompositions, so the total number of update operations required to reconcile the change is less.

Convolution and Reduced Convolution. The first method proposed in this paper identifies critical scale factors for each edge in the convolution and updates the arrangement of the convolution according to the critical scale factors. The second method proposed in the paper performs dynamic updates on the convolution without computing critical events. Our methods can be used in any convolution-based method, such as $[30,3,4]$. However, to ease our discussion, we will introduce our method based on the reduced convolution approach presented in our previous work $[23,8]$, which we review briefly here. In order to compute the convolution of two shapes, $P$ and $Q$, we consider an edge $e$ of $P$ and a vertex $v_{i}$ of $Q$. We say that $e$ is compatible with $v_{i}$ if the direction of $e$ 's outward normal is "between" the directions of the outward normals of $v_{i}$ 's incident facets.

In 2D, we assume $P$ and $Q$ are both counter-clockwise oriented polygons. Then an edge $e$ and a vertex $v$ are compatible if the direction of $e$ 's outward normal is between those of $v$ 's incident edges. Note that, this is equivalent to say that if the direction of $e$ is on the counter-clockwise turn from $\left(v_{i-1}, v_{i}\right)$ to $\left(v_{i}, v_{i+1}\right)$. See Fig. 2 for an example in $2 \mathrm{D}$.

In $3 \mathrm{D}, f$ and $v_{i}$ are compatible if the outward normal of $f$ is within the region of the Gaussian map enclosed by the outward normals of $v_{i}$ 's incident facets. Briefly, the 
Gaussian map is a mapping of a 3D surface to a unit sphere. In the case of polyhedral models, each face is mapped to a point on the sphere's surface equal to its unit normal. These mapped points are connected by edges if their associated faces on the model are adjacent-this maps the edges of the model to edges in the Gaussian map. Finally, these point/edge mappings create regions on the surface of the sphere which are the mappings of the vertices on the surface of the model.

If $f$ and $v_{i}$ are compatible, then the facet $f+v$ (called $f v$-facet) appears in the convolution. Faces of the convolution in 3D may also be formed by an edge from $P$ and an edge from $Q$ (called $e e$-facet). In this case, the edges are compatible if their associated edges in the Gaussian map intersect, in which case the facet $e_{P} \oplus e_{Q}$ appears in the convolution.

In order to compute the reduced convolution ( $\mathrm{RC})$, we compute only a subset of the convolution. We exclude contributions from reflex vertices (2D) and edges (3D). It has been proven that contributions from reflex vertices and edges are guaranteed to be interior to the Minkowski sum [8]. To extract the Minkowski sum from the reduced convolution, intersections between the edges and facets of the reduced convolution are computed. Then properly nested orientable loops (2D) and shells (3D) are extracted from the intersections of the RC. Finally, a collision detection step is used to detect any remaining errant loops and shells. The worst-case complexity of both algorithms is $O\left(m^{d} n^{d}\right)$, for $P, Q \subset \mathbb{R}^{d},|P|=m,|Q|=n$, which is optimal with respect to the worst-case complexity of the Minkowski sum boundary. However, experimentally, our method is shown to be much faster than Wein's [30].

\section{Uniform Scaling in 2D}

We seek to update the Minkowski sum of polygons $P$ and $Q$ transforming under uniform scales. We assume without loss of generality that $P$ scales uniformly and $Q$ remains fixed. Since the Minkowski sum is commutative, scaling $Q$ can be done identically by simply swapping $P$ and $Q$ in the input order. The uniform scaling operation $P^{\prime}=s P$ generates $P^{\prime}$ such that for every point $v \in P, s v \in P^{\prime}$. A key observation is that when $P$ scales by $s$, the outward normals of all its line segments remain the same, and so scaling of $P$ does not change the combinatorial structure of the reduced convolution ( $\mathrm{RC}$ ); only the length and position of the segments in $\mathrm{RC}$ change.

Even though the combinatorial structure of the RC does not change when $P$ is scaled, the outer boundary of the Minkowski sum will change. In particular, the set of edges (2D) or facets (3D) which intersect with each other may change-some intersections may be deleted while others may be added. We call these additions and deletions critical events.

To use these critical events, we compute the ranges of $s$ within which intersections between edges actually occur. We can then add or delete intersections by stepping through the critical events between two values of $s$. When we finish adding and deleting intersections in this way, we can correctly and efficiently update by considering only those

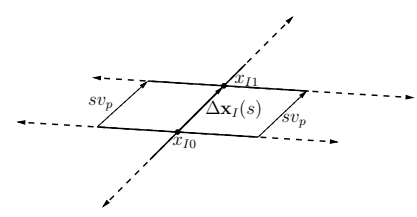

(a) T-edge

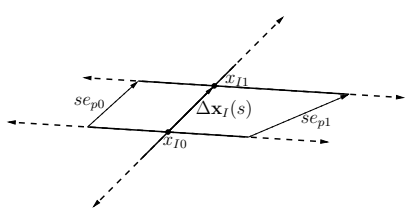

(b) S-edge
Fig. 3. In (a) an edge from $Q$ and a vertex from $P$ form a T-edge in the convolution, so the segment only translates as $s$ changes. In (b) an edge from $P$ and a vertex from $Q$ form an S-edge edge, so the segment also scales as it translates. In both cases the underlying lines translate.

intersections between line segments that are valid for the given $s$.

\subsection{Tracking the intersection values in $2 D$}

Consider a single edge of the convolution, $a$. Suppose $a$ is formed from the contribution of an edge of $P, e_{p}$, and a vertex of $Q, v_{q}$. Then $a=e_{p}+v_{q}$, that is, $e_{p}$ translated by $v_{q}$. When $P$ scales, only $e_{p}$ changes, so $a(s)=s e_{p}+v_{q}$. Because such edges are scaled as $P$ is scaled, we call them S-edges. Note that S-edges will also be translated in the updated convolution. Similarly, if $a$ is formed from an edge of $Q, e_{q}$ and a vertex of $P, v_{p}$, then $a=e_{q}+v_{p}$, and $a(s)=$ $e_{q}+s v_{p}$, since only $P$ scales. We call such edges T-edge since as $P$ is scaled, they are translated but not scaled. Fig. 3 illustrates both types of edges.

Now consider two line segments $a$ and $b$ in the convolution, and let their endpoints be $\left(a_{0}, a_{1}\right)$ and $\left(b_{0}, b_{1}\right)$, respectively. Let the underlying lines of $a$ and $b$ be $l_{a}$ and $l_{b}$, respectively. We parameterize the intersection point of $l_{a}$ and $l_{b}$ over $s \in(0, \infty)$. To do this, we consider the parameterized forms of $l_{a}$ and $l_{b}$ :

$$
\begin{aligned}
& l_{a}(r)=a_{0}+r\left(a_{1}-a_{0}\right)=a_{0}+r \mathbf{v} \\
& l_{b}(t)=b_{0}+t\left(b_{1}-b_{0}\right)=b_{0}+t \mathbf{w},
\end{aligned}
$$

where $\mathbf{v}=a_{1}-a_{0}$ and $\mathbf{w}=b_{1}-b_{0}$.

Observation 1 Let $\boldsymbol{u}=a_{0}-b_{0}$. Then the intersection point of $l_{a}$ and $l_{b}$ is at

$$
r_{I}=\frac{\boldsymbol{v}_{y} \boldsymbol{u}_{x}-\boldsymbol{v}_{x} \boldsymbol{u}_{x}}{\boldsymbol{v}_{x} \boldsymbol{w}_{y}-\boldsymbol{v}_{y} \boldsymbol{w}_{x}}
$$

and the coordinate of that intersection is $x_{a}\left(r_{I}\right)=a_{0}+r_{I} \boldsymbol{v}$.

The parameterized form lets us find the critical events we are looking for.

\subsection{Finding critical regions}

Let $x(s)$ be the intersection point between the line $l_{a}$ containing $a$ and the line $l_{b}$ containing $b$ when $P$ is scaled by a factor of $s . x(s)$ is a parameterized line as well. Let the intersection point computed above be $x_{0}$. This intersection is computed at the base scale value, $s=1$. Therefore, $x(1)=x_{0}$. Then we can define $x(s)=x_{0}+(s-1) \sigma$ for a slope vector $\sigma$. It is easy to recompute the line segments at some other scale, $s^{\prime}$, and compute the intersection of the new lines to find $x\left(s^{\prime}\right)=x_{0}+\left(s^{\prime}-1\right) \sigma$, and so $\sigma=\frac{x\left(s^{\prime}\right)-x_{0}}{s^{\prime}-1}$. We choose $s^{\prime}=2$, since in this case, $s^{\prime}-1=1$ and so $\sigma=x(2)-x(1)$, which eliminates the division. 
Assume that $a$ and $b$ that do not intersect. As $P$ scales, if $a$ and $b$ start to intersect, they must do so first at an endpoint of either $a$ or $b$. Similarly, when $a$ and $b$ separate, their final point of contact will be at an endpoint of either $a$ or $b$. The values of $s$ where these initial and final points of contact for some set of edges occur are the critical events.

We begin by parameterizing the endpoints of $a$ over $s$, as $a_{0}(s), a_{1}(s)$. We wish to find the values of $s$ for which $x(s)=a_{0}(s)$ and $x(s)=a_{1}(s)$ that is, the scale factors for which the intersection of the two edges is precisely the endpoint of one of them.

\subsubsection{Critical region for $S$-edge}

Suppose that $a$ is an S-edge in the convolution is formed from an edge, $e_{p}$ in $P$ and a vertex $v_{q}$ in $Q$. Then:

$$
\begin{aligned}
& a_{0}(s)=e_{p 0} s+v_{q} \\
& a_{1}(s)=e_{p 1} s+v_{q}
\end{aligned}
$$

Setting $x(s)=a_{0}(s)$ we obtain $x_{0}+(s-1) \sigma=s e_{p 0}+v_{q}$, $x_{0}-\sigma-v_{q}=s\left(e_{p 0}-\sigma\right)$. This is an equation of the form: $s \alpha=\beta$, where $\alpha=\left\langle\alpha_{1}, \alpha_{2}\right\rangle$ and $\beta=\left\langle\beta_{1}, \beta_{2}\right\rangle$. Since $s$ is scalar in uniform scaling, this is an overdetermined system of equations; there is no solution for $s$ if the system is inconsistent. Otherwise, we obtain a possible bound on $s$ values for contact with the $a_{0}(s)$. We can proceed to totally bound the $s$ interval where the intersection lays on the segment $a$ by computing the same bound for $a_{1}(s)$, which is of the same form.

\subsubsection{Critical region for T-edge}

But what about convolution edges formed from an edge $e_{q}$ of $Q$ and a vertex $v_{p}$ of $P$ ? Assume, without loss of generality, that $b$ is such an edge, and so $b=e_{q}+v_{p}$. Then its endpoints are defined by $b_{0}(s)=e_{q 0}+s v_{p}, b_{1} s=e_{q 1}+s v_{p}$.

Again we seek to bound the intersection in this case by finding $s$ such that $x(s)=b_{0}(s)$ and $x(s)=b_{1}(s)$. As above, $x(s)=x_{0}+(s-1) \sigma$, and so the form of these equalities are: $x_{0}+(s-1) \sigma=e_{q i}+s v_{p}$. This is an overdefined system of the same form as above, subject to the same results.

\subsubsection{Critical region of a segment pair}

To obtain the intersection interval for the segment pair, we just take the intersection of the intervals computed above, $\left[\max \left\{B_{a_{0}}, B_{b_{0}}\right\}, \min \left\{B_{a_{1}}, B_{b_{1}}\right\}\right]$ where $B_{u}$ is the $s$ boundary for the endpoint $u$.

We can compute these intervals for all pairs of line segments as a pre-processing step in $O\left(n^{2}\right)$. Using this, we can compute a list of critical events $-s$ values when intersections are introduced into the arrangement or removed from it. This allows us to update only those intersections at each scaling operation which are relevant. Because computing the intersections of the arrangement is the largest bottleneck in the reduced convolution method, this promises significant speed advantages over recomputing the arrangement from scratch every time $s$ changes.

\subsubsection{Update arrangement for a given scale factor $s$}

In order to update the arrangement, we produce an array of critical events: pairings of $s$ values with a list of insertions or deletions since the last event produced by the intervals for pairs of line segments. Given an initial scale value $s_{0}$ and its arrangement, and a final scale value $s_{\text {final }}$, we can step through the structure, deleting and adding nodes in the arrangement as necessary at each event until we reach an event such that $s_{\text {event }}>s_{\text {final }}$, at which point we update all of the remaining nodes according to the above equations.

\section{Uniform scaling in $3 \mathrm{D}$}

In $2 \mathrm{D}$, events are defined by the contact of two edges. Similarly, in 3D, events are defined by the contact of two faces. There are two possible cases for these events in 3D: a vertex of one face comes into contact with any part of another face, or an edge of one face comes into contact with an edge of the other face without any vertex contacts. We call these vertex events and edge events, respectively.

\subsection{Finding vertex events}

Computing vertex events is a relatively straightforward extension of the $2 \mathrm{D}$ case. Given two faces, $f_{1}$ and $f_{2}$, we consider each edge $e$ of $f_{1}$ independently, and we parameterize the line $l$ underlying $e$ and the plane $p$ underlying $f_{2}$, and compute the intersections at $s=1$ and $s=2$ as in the $2 \mathrm{D}$ case. Uniform scaling still only causes linear motion in $3 \mathrm{D}$, and so computing where the vertex contact occurs is fundamentally identical to the $2 \mathrm{D}$ case.

We can again compute $\sigma=x(2)-x(1)$, and parameterize the intersection point across $s$ as $x(s)=x_{0}+(s-1) \sigma$. We then set the intersection point equal to the parameterized endpoints $e_{1}(s)$ and $e_{2}(s)$ to find candidate events, identically to the $2 \mathrm{D}$ case. The equation $x(s)=e_{i}(s)$ is overdetermined. There are three equations and only one free variable, similar to the $2 \mathrm{D}$ case, and so there may be no consistent solution again - this will occur when the edges are parallel or skew.

If there is a consistent solution we check and make sure these intersection points also lay in the face $f_{2}$ just as one would ensure that the contacts in 2D lay on the line segment and not just the line. The vertex events between the edges of $f_{2}$ and $f_{1}$ are found in the same way.

\subsection{Finding edge events}

Computing edge events is slightly more complicated. We consider two edges, $e_{1}$ and $e_{2}$, and their underlying lines, $l_{1}$ and $l_{2}$ respectively. We cannot simply check for an intersection point since it is most likely that $l_{1}$ and $l_{2}$ are skew. Instead, faces $f_{1}$ incident to $e_{1}$ and $f_{2}$ incident to $e_{2}$, and their supporting planes, $p_{1}$ and $p_{2}$ respectively. We are interested in the intersection $x_{1}$ between $l_{1}$ and $p_{2}$ and the intersection $x_{2}$ between $l_{2}$ and $p_{1}$. Then $l_{1}$ and $l_{2}$ will intersect precisely when $x_{1}=x_{2}$.

A line $l$ which underlays an edge $e$ with endpoints $e_{a}$ and $e_{b}$ can be parameterized as $e_{a}+\left(e_{b}-e_{a}\right) t$, and the plane 
$p$ which underlies triangle $t$ with vertices $t_{a}, t_{b}, t_{c}$ can be parameterized as $t_{a}+\left(t_{b}-t_{a}\right) u+\left(t_{c}-t_{a}\right) v$. We set these equal to each other and simplify, yielding

$$
e_{a}-t_{a}=\left[\begin{array}{c}
e_{a}-e_{b} \\
t_{b}-t_{a} \\
t_{c}-t_{a}
\end{array}\right]^{T}\left[\begin{array}{l}
t \\
u \\
v
\end{array}\right]
$$

We have five vertices from the convolution in this equation, $e_{a}, e_{b}, t_{a}, t_{b}, t_{c}$, each of which is parameterized in the scale domain by its $P$ and $Q$ components, $v=v_{q}+s v_{p}$. Substituting, we obtain expressions for $t, u$ and $v$ parameterized by s:

$$
\left[\begin{array}{c}
t \\
u \\
v
\end{array}\right]=\left(\left[\begin{array}{c}
e_{a q}-e_{b q}+s\left(e_{a p}-e_{b p}\right) \\
t_{b q}-t_{a q}+s\left(t_{b p}-t_{a p}\right) \\
t_{c q}-t_{a q}+s\left(t_{c p}-t_{a p}\right)
\end{array}\right]^{T}\right)^{-1} \quad\left(e_{a q}-t_{a q}+s\left(e_{a p}-t_{a p}\right)\right)
$$

When we solve this for $l_{1}$ and $p_{2}$ as well as $l_{2}$ and $p_{1}$, we obtain two expressions in $s$ for the intersections of the line and the plane, which we can set equal to each other and solve for $s$.

Parallel faces When $f_{1}$ and $f_{2}$ are parallel but not always coplanar, then

$$
\left[\begin{array}{c}
e_{a}-e_{b} \\
t_{b}-t_{a} \\
t_{c}-t_{a}
\end{array}\right]^{T}
$$

will be singular, and so we will not

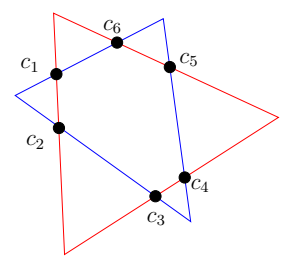

Fig. 4. Degenerate $e e$ contacts for parallel faces, contacts are marked as $c_{1}$ through $c_{6}$. Notice that no vertices are involved in this event. be able to detect edge events in the usual way. If any vertex of either face is involved in the contact, then we will correctly detect these events as vertex events, but it is possible that this isn't the case. Figure 4 shows a situation in which two coplanar faces have no vertex contacts. In order to determine whether an event will occur, we must find the scale value when the faces are coplanar, and then check the edges of the faces for intersections at that scale. Fortunately, the detection for vertex events is robust enough that it does not need to deal with parallel faces as a special case.

To determine the value of $s$ where the faces are coplanar, we pick an arbitrary vertex $v$ on $f_{1}$ and proceed as though we were detecting a vertex event with $f_{2}$. However, we do not need to check if $v\left(s_{\text {event }}\right)$ is inside of $f_{2}$, since we are only interested in the value of $s$ where $v$ hits $p_{2}$-this will be the value of $s$ at which the faces are coplanar.

Because of this, we do not need to perform the usual edge event detection at all when dealing with parallel faces. For these faces, we find the scale of contact from the vertex event detection step. If there are no vertex events, we check for edge intersections to see if there are any edge events.

Coplanar faces Finally, in some cases, $f_{1}$ and $f_{2}$ will always be coplanar. In these coplanar cases, the contact possibilities for $f_{1}$ and $f_{2}$ reduce to the $2 \mathrm{D}$ case, in which edge events are impossible. Therefore, we can use the logic from the $2 \mathrm{D}$ scaling directly in order to detect the events for the coplanar faces by transforming $f_{1}$ and $f_{2}$ into the $x y$-plane, and applying $2 D$ vertex event detection.

\section{Non-uniform scaling for convex polyhedra}

In three dimensions, the worst-case complexity of the event space is $O\left(m^{4} n^{4}\right)$. The memory necessary to store the event structure and the time necessary to compute it rapidly become overwhelming. While there are several practical strategies for mitigating memory issues, including narrowing the scaling domain to a smaller window and disk swapping, the event space must be computed for all pairs of faces, resulting in worst-case time complexity. Enumerating all critical events also makes the extension to nonuniform scaling almost impossible. As a result, we look to a method with less pre-computation.

Similar to our strategy for handling rotation [7], we propose to dynamically repair the Minkowski sum after scaling. To do so, we define errors in the transformed convolution to be convolution facets constructed from either $v f$-pairs or $e e$-pairs that are no longer compatible, called $f v$-errors and $e e$-errors, respectively ( $f v$ - and $e e$-facets are defined in Section 3). Our method works simply by correcting the errors introduced by the scaling. Correcting the $f v$-errors involves gradient descent on the degree of incompatibility until the vertex and face pair become compatible. Let $f$ be a facet of $P$ and $v$ be a vertex of $Q$. When $f$ and $v$ are compatible, all the edges that are incident to $v$ must be below or on the half-plane supported by $f$. When $f$ and $v$ are incompatible, we can define the degree of incompatibility: $I C(f, v)=\max \left\{\mathrm{d}(e, f) \mid e \in E_{v}\right\}$, where $E_{v}$ is a set of edges incident to $v$ and $\mathrm{d}(e, f)$ is the longest Euclidean distance from any point on $e$ to $f$. We say an edge $e$ is the witness of the incompatibility if $\mathrm{d}(e, f)$ is $I C(f, v)$. Fig. 5(a) illustrates an example of $f v$-error and $I C(f, v)$.

It is not difficult to show that any ee-errors must coexist with $f v$-errors (see Theorem 1). Since, for convex pairs, there are only $O(m+n) v f$-facets, but $O(m n) e e$-facets, we can increase the repairing speed by first checking all $f v$ facets for errors. Using the $f v$-errors as starting points, we then form chains of ee-errors connected to the $v f$-error.

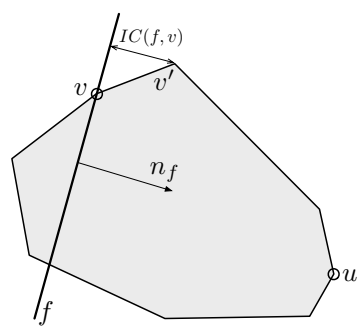

(a) degree of incompatibility

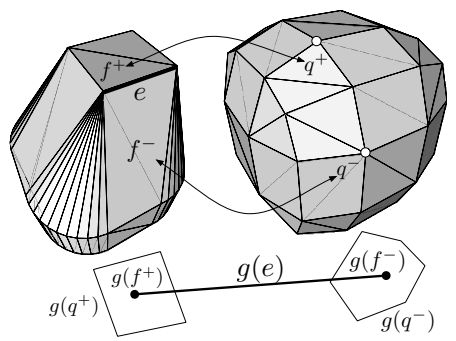

(b) correct an ee-error
Fig. 5. (a) A 2-d drawing shows the definition of $I C(f, v)$ and its witness $\overline{v v^{\prime}}$. Using gradient decent, we will find the compatible vertex $u$ for $f$. (b) Determine the associated edges for edge $e$.

\section{Theorem $1 f v$-errors and ee-errors must coexist.}

PROOF. Let $g(p)$ be the Gaussian map of a primitive $p$. We first show that if there is an ee-error, there must be an $f v$-error. Let $e$ and $e^{\prime}$ be a pair of edges that are compatible before scaling and become an ee-error after scaling. When $e$ and $e^{\prime}$ are compatible, $g(e)$ and $g\left(e^{\prime}\right)$ must intersect and, after a non-uniform scaling of $P, g(e)$ and $g\left(e^{\prime}\right)$ no longer 
intersect. This means at a certain point during the scaling, an end point of $g(e)$ must cross $g\left(e^{\prime}\right)$ or vice versa. When a point $v$ crosses the edge $g(e), v$ changes the face with which it is associated from one side of $g(e)$ to the other side of $g(e)$. This change indicates that there must be an $f v$-error.

We then show that if there is an $f v$-error, there must be an ee-error. If a facet $f$ of $P$ and a vertex $v$ of $Q$ become an $f v$-error, we know that $f$ now must be compatible with some other vertex $v^{\prime} \neq v$ of $Q$. As a result, an edge $g(e)$ incident to $g(f)$ must be moved (or deformed) with $g(f)$. Since the faces in $g(Q)$ are convex and $g(e)$ cannot intersect with a segment more than twice, $g(e)$ must intersect with some new edges of $Q$ when $g(f)$ moves from $g(v)$ to $g\left(v^{\prime}\right)$. This indicates that there must be at least one ee-error.

Therefore, $f v$-errors and ee-errors must coexist.

More specifically, let $e$ be such an edge from $P$ involved in an ee-error, and let $f^{-}$and $f^{+}$be the facets in $P$ incident to $e$. Assume that the facets $f^{-}$and $f^{+}$both have the compatible vertices $q^{-}$and $q^{+}$of $Q$. If we overlay the Gaussian map $g(e)$ of $e$ with $g(Q), g(e)$ will intersect a set of faces in $g(Q)$ and the end points of $g(e)$ are inside $g\left(q^{-}\right)$and $g\left(q^{+}\right)$. See the bottom of Fig. 5(b). If we can determine the rest of the faces intersected by $g(e)$, we can find the compatible edges for $e$. We further know that these faces form a connected component between $g\left(q^{-}\right)$and $g\left(q^{+}\right)$, thus the compatible edges for $e$ must be on the boundary of these faces. To find these Gaussian faces, we start from $g\left(q^{-}\right)$, and find an incident edge $e^{\prime}$ of $g\left(q^{-}\right)$that is compatible with $e$.

Because both $P$ and $Q$ are convex, the faces $f_{1}$ and $f_{2}$ incident to $e$ will each be compatible with precisely one vertex, $v_{1}$ and $v_{2}$ respectively. The edges with which $e$ is compatible must form a path from $v_{1}$ to $v_{2}$ along the surface of the model. We check each of $v_{1}$ 's incident edges to find the edge with which $e$ is compatible. We then replace $v_{1}$ with the vertex at the other end-point of the compatible edge, and repeat, ignoring the incident edge which has already been found to be compatible. When we find $v_{2}$ by this path, we have enumerated all of the compatible vertices. The complexity of this update is $O(|E|)$, where $E$ is the set of edges incident to at least one vertex in the path.

In convex pairs, each face of $P$ will be paired with precisely one vertex of $Q$ and vice-versa. We depend on this relationship in order to correctly identify errors. An extension to non-convex inputs depends on the existence of a convex map-a convex polyhedron whose Gaussian map is identical to the arrangement of the Gaussian map of the non-convex input. Discussion of the convex map is outside the scope of this paper.

\section{Results}

Our implementations are based on the publicly available implementation from our previous work in 2D [8] and $3 \mathrm{D}[7]$. All experiments reported in this section are performed on a machine with Intel CPUs at $2.13 \mathrm{GHz}$ with 4 GB RAM and our implementation is coded in $C++$, using GNU MPFR and the MPFRC++ wrapper for high precision arithmetic.

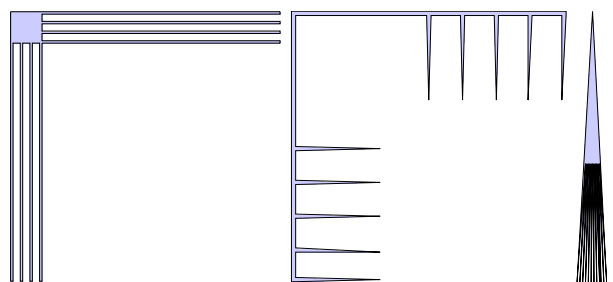

(a) G1 (30) $\quad$ (b) G2 (34) $\quad$ (c)

G3

(24)

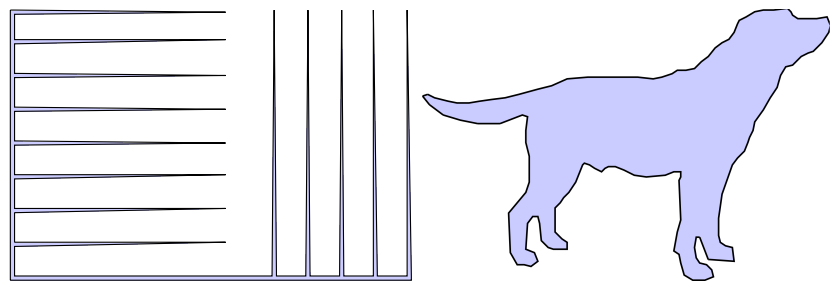

(d) G4 (43)

(e) $\operatorname{dog}(145)$

Fig. 6. Models used in the experiments. The figure shows the names and the sizes of the polygons. Note that (c) is G3 (24).

\subsection{Results from uniform Scaling in 2D}

Table 1

Average speed-up of the $2 \mathrm{D}$ enumerative dynamic update algorithm over 100 random re-scalings. Models in the first column are the model being scaled in each experiment, while models in the first row are the static (unscaled) model. The bolded figures are the lowest and highest speed-up values achieved.

\begin{tabular}{|c||c|c|c|c|c|}
\hline & $d o g$ & $g 1$ & $g 2$ & $g 3$ & $g 4$ \\
\hline$d o g$ & 28.911 & 115.651 & $\mathbf{5 . 5 9 2}$ & 32.408 & 6.538 \\
\hline$g 1$ & 115.651 & 131.150 & 432.069 & 533.141 & $\mathbf{4 7 3 . 2 8 2}$ \\
\hline$g 2$ & 15.223 & 313.710 & 298.038 & 161.712 & 88.916 \\
\hline$g 3$ & 112.354 & 152.790 & 171.825 & 34.347 & 79.362 \\
\hline$g 4$ & 14.519 & 201.861 & 45.220 & 80.230 & 122.565 \\
\hline
\end{tabular}

Table 1 shows average speed-up factors for several model pairs in Fig. 6 across the scaling domain $[0.1,10]$ in increments of 0.1 scale factor. Speed-up is computed as $\frac{t_{\text {static }}}{t_{\text {dynamic }}}$ over 100 random re-scalings. Our method is always faster than recomputing from scratch and is on average 150.682 times faster. The largest speedup is over two orders of magnitude (from $g 1 \oplus g 4$ ) and even on model pairs with high event density ( $\operatorname{dog} \oplus g 2$ in Table 1$)$, the proposed method is faster though the improvement here is more marginal.

The results in Fig. 7 show speed-up factors on one of the more complex model pairs, shown in Figs. 7(a) and 7(b). The monkey model contains 1204 vertices, and the bird model has 275 vertices. Their reduced convolution contains 16,425 segments. When the monkey is scaled along the interval $s \in(0, \infty]$, there are $56,996,430$ critical events.

Fig. 8 shows speed-up factors for the neuron $\widehat{\otimes}$ disc pair shown in Fig. 1. There are 1815 vertices in the neuron model and 32 vertices in the disc model. The reduced convolution of the neuron and the disc has 3196 segments. There are 56238 critical events discovered when the disc is scaled across $s \in(0, \infty)$. 

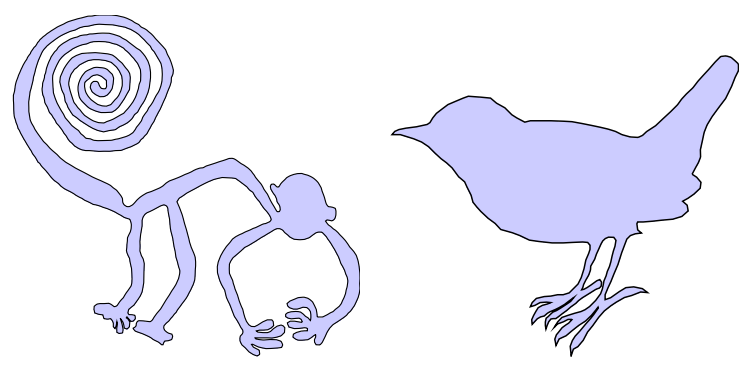

(a) Input $P$, monkey (1204)

(b) Input $Q$, bird (275)

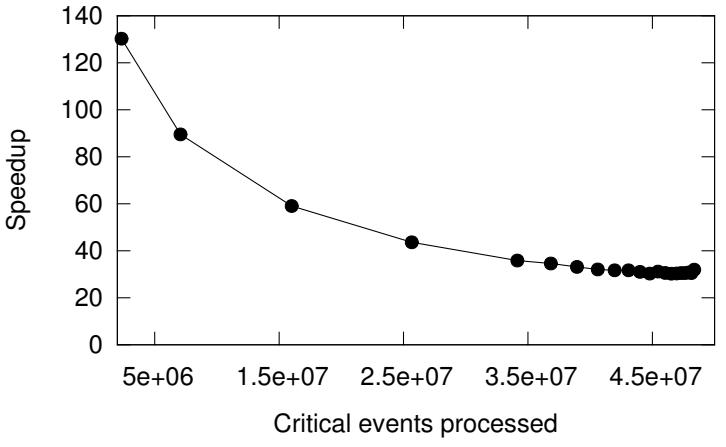

(c) Speed-up vs. Number of critical events processed

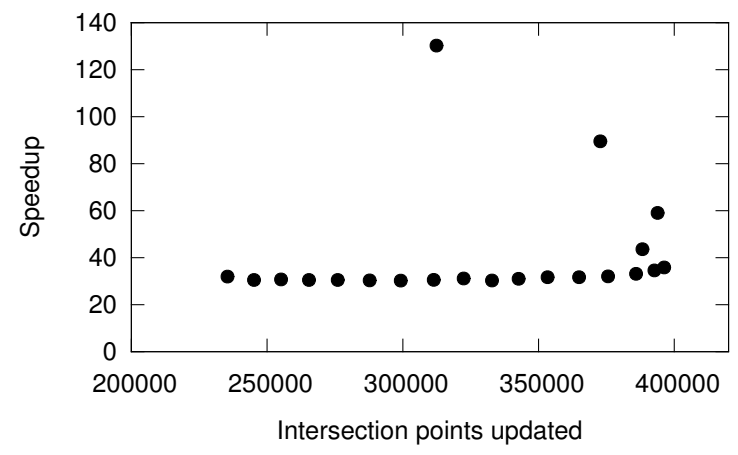

(d) Speed-up vs. Number of intersections updated

Fig. 7. Speed-up as a function of critical events and number of intersections updated as $P$ scales in $3 \mathrm{D}$. The number of events processed is a much better predictor of the speed gain than the number of intersections updated, as events generally outstrip intersectins in number by orders of magnitude.

For more complex model pairs, the table shows that the update method outperforms recomputing the intersections by an order of magnitude in general. Of course, for larger scale jumps, the bottleneck of this method is in updating the events as $P$ scales. This is largely due to the high number of events that occur, especially in complex model pairs. In practice, however, scaling that needs to be done continuously tends to occur over smaller intervals that are generally quick to update.

The time to compute the scale bounds in $2 \mathrm{D}$ is on the order of the time necessary to compute the intersections using brute force. Asymptotically, the time bound of $O\left(n^{2}\right)$ for the initial computation to identify all intersections and all events is worse than the best-case bound for the static computation of intersections, $\Theta(n \log n)$ (with a constant number of intersections). However, on average, the time taken to compute the events during dynamic scaling is a

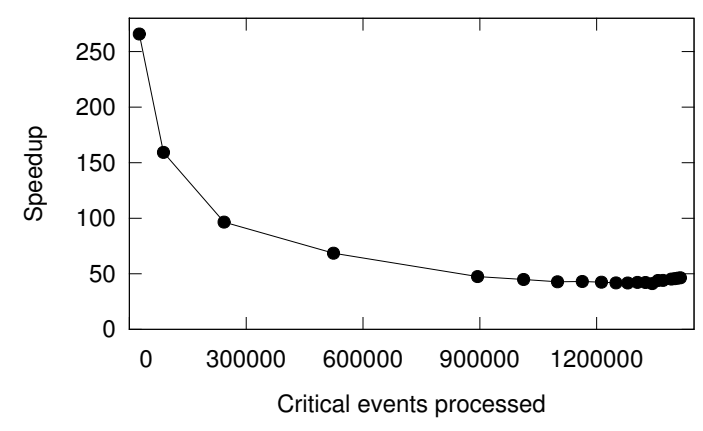

(a) Speed-up vs. Number of critical events processed

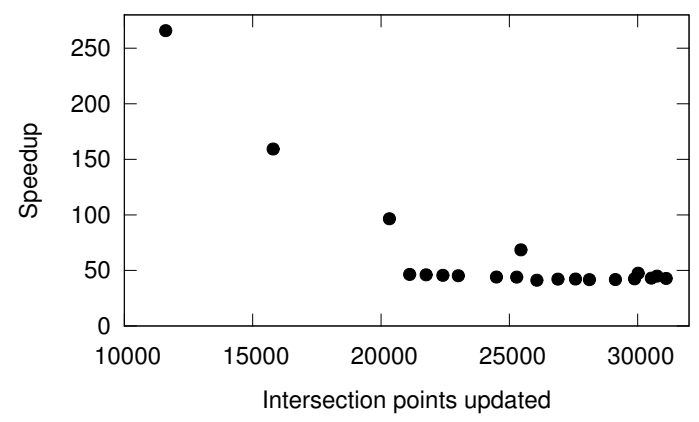

(b) Speed-up vs. Number of intersections updated

Fig. 8. Speed-up factors for the neuron $\widehat{\otimes}$ disc pair from Fig. 1. Again, number of events processed is a good predictor for speed-up, while number of intersections processed is not, though in this case it correlates more consistently than in monkey $\widehat{\otimes}$ bird. (a) Speed-up by critical events for neuron $\widehat{\otimes}$ disc. (b) Speed-up by intersections updated for neuron $\widehat{\otimes}$ disc.

fraction of the time taken to compute the intersections. The monkey/bird model pair required on average about 140 seconds to build the initial intersections, but only about 28.7 seconds to compute the events. The neuron/circle pair took about 5.3 seconds to compute its nodes, but only 1.25 seconds to compute the events.

Even though both pre-processing steps are asymptotically similar, on average the time taken to compute the events is a fraction of the time taken to compute the intersections. The monkey/bird model pair required on average about $140 \mathrm{~s}$ to build the initial intersections, but only about $28.7 \mathrm{~s}$ to compute the events. The neuron/disc pair took about $5.3 \mathrm{~s}$ to compute its nodes, but only $1.25 \mathrm{~s}$ to compute the events.

With that in mind, given significant speed-up from our method only a small number of queries are needed to make up the difference in pre-computation time before it is vastly more expensive to perform the entire Minkowski sum computation again and again. Additionally, in this case it is reasonable to treat the initial computation of intersections as a pre-computation for situations requiring dynamic scaling queries. The advantage of the brute-force method then is it simplify in implementation because there are fewer degeneracies to account for. 


\subsection{Results from Uniform and Non-Uniform Scaling in 3D}

\subsubsection{D enumerative scaling under uniform scaling}

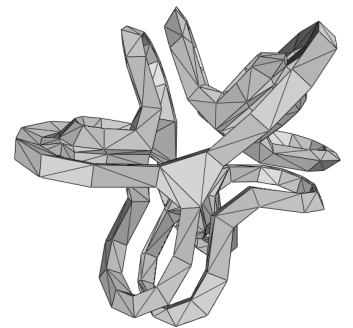

(a) knot, 992 facets

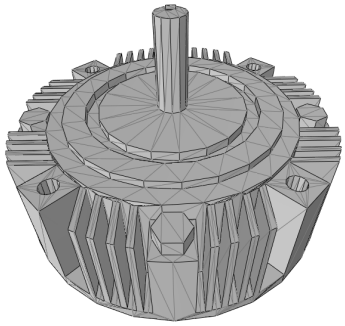

(b) clutch, 2116 facets

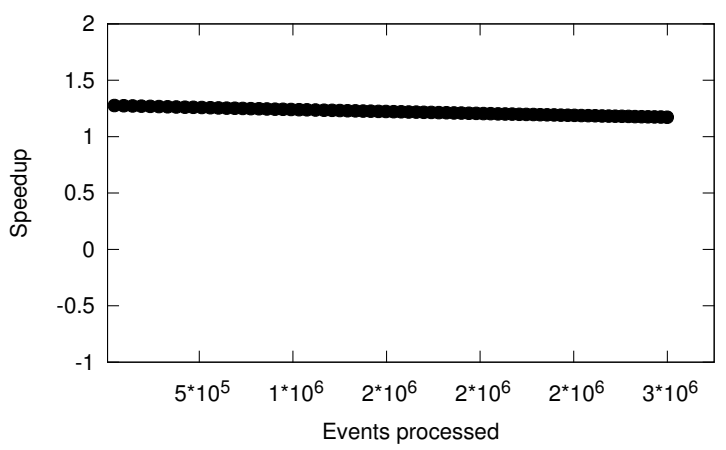

(c)

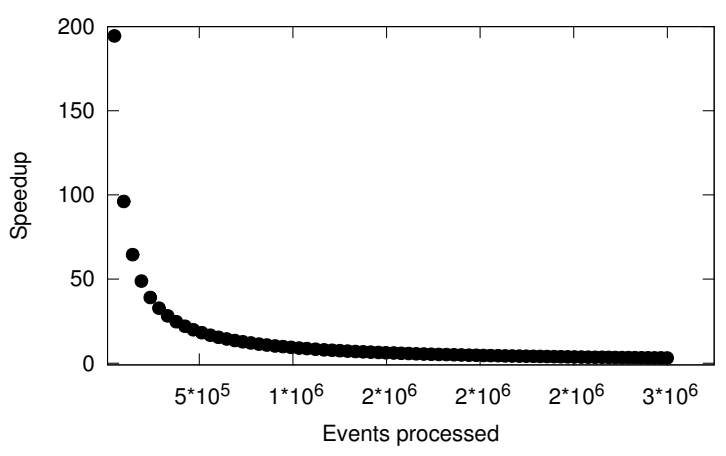

(d)

Fig. 9. (a) Speed-up from updating knot $\oplus$ clutch using event enumeration, including time used for collision detection. (b) Speed-up for updating intersections only.

The enumerative approach does not scale well in 3D. In Fig. 9, we see speed-up values for the $k n o t \oplus$ clutch model pair by number of events processed. Computing the event structure took approximately 65 minutes, and computed $8,427,881$ events on the scaling domain $[0.2,2]$. We see a similar asymptotic behavior in speed-up over recomputing the line intersections from scratch, as we do in 2D. However, unlike $2 \mathrm{D}$ examples, the collision detection step that we use to filter out boundaries interior to the Minkowski sum boundary takes about $50 \%$ of the total computation time [23]. When we compute total overall speedup, the enumerative method does not provide a significant increase in speed, especially considering the extremely long time needed to compute the event structure.

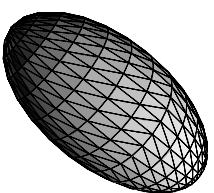

(a) (b)

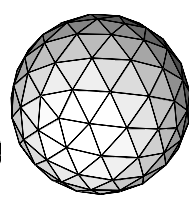

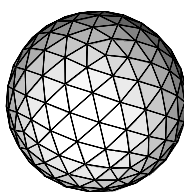

(c)

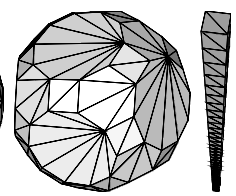

(d) (e)
Fig. 10. Models used in the experiments: (a) ellipse, 960 facets, (b) GS3, 320 facets, (c) GS4, 500 facets, (d) truncated icosidodecahedron (TI), 236 facets, (e) v-rod, 324 facets.

\subsubsection{Non-uniform scaling for convex polyhedra}

We tested the 3D non-enumerative update-based method against computation from scratch over 100 random nonuniform scaling factors. Note that we have no experimental results for convex models in the previous sections because scaling the convex models uniformly simply makes their Minkowski sums scale uniformly. On the contrary, non-uniform scaling provides more complex and interesting behaviors to the Minkowski sums of convex models.

Our results for non-uniform scaling using models in Fig. 10 are shown in Table 2. The speedup values shown, computed again as $\frac{t_{\text {static }}}{t_{\text {dynamic }}}$, are the average speed-up over 100 queries. Here, we see significant speed-up (between 13 and 94) for computing convex Minkowksi sums dynamically over manual recomputation across all studied models. Note these figures are much less varied than the 2D case because the the convolution for convex pairs is always equal to the Minkowski sum boundary, and so no culling is necessary.

Table 2

Average speed-up of the 3D non-enumerative dynamic update algorithm over 100 random non-uniform rescalings. Models in the first column are the model being scaled in each experiment, while models in the first row are the static (unscaled) model. There is no collision detection component here, the values provided are total speed-up. The bolded values are, again, the lowest and highest speed-up values achieved.

\begin{tabular}{|c||c|c|c|c|c|}
\hline & ellipse & $G S 3$ & $G S 4$ & $T I$ & v-rod \\
\hline ellipse & 17.231 & 16.653 & 32.312 & 14.636 & 19.060 \\
\hline$G S 3$ & 44.475 & 18.311 & 17.584 & 17.423 & 29.902 \\
\hline$G S 4$ & $\mathbf{1 3 . 1 0 9}$ & 17.273 & 13.050 & 18.026 & 34.342 \\
\hline$T I$ & 18.685 & 20.332 & 19.669 & 24.952 & 37.960 \\
\hline v-rod & 24.471 & 40.246 & 40.691 & 44.757 & $\mathbf{9 7 . 4 1 0}$ \\
\hline
\end{tabular}

\section{Applications}

In this section, we discuss two direct applications of the proposed dynamic Minkowski sum method.

\subsection{Motion planning and rapid virtual prototyping}

Our method has applications to motion planning problems with repeated, scaled objects. As shown in Fig. 11, the example environment contains only 12 scaled objects yet has a speed-up factor of 38.8 over recomputing the intersections from scratch. Very few scaling requests are necessary to make up the difference caused by pre-processing 


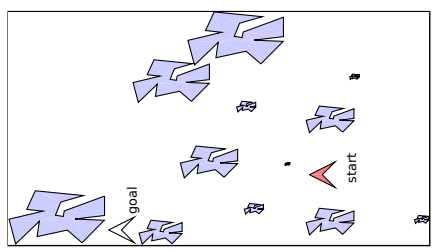

(a) Example environment

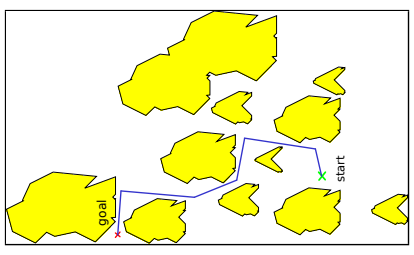

(b) Corresponding C-space
Fig. 11. An example environment with repeated scaled obstacles. In (a), unfilled polygons are the obstacles, the filled polygon is the robot. Computing the convolution intersections for the example environment required $96.811 \mathrm{~ms}$ recomputing the intersections from scratch, but only $2.498 \mathrm{~ms}$ using our update method, a speed-up factor of approximately 38.755.

the events. As a result, the efficiency of computing configuration spaces for motion planning environments is greatly enhanced.

Furthermore, the method can also be used for a robot of uncertain size to answer the following query: "Given a base assumed size for the robot, what is the largest scale factor for which a given path is valid?" by computing events for the robot's scaling. Similar to the contact point computation for intersection events, such events can be computed for segments of the robot's path. This allows reporting of the scale of first contact between an obstacle boundary and the robot's path. In fact, computing these events is easier than computing the convolution events, because the robot's path in this case does not vary with scale.

In rapid prototyping, it is important to minimize waste of both time and materials by guaranteeing that the parts of any assemblies produces will fit together once they are constructed from the CAD input. However physical objects are manufactured to tolerance, while CAD models do not have this limitation. The models can clip through each other, or produce physical output that is larger than the virtual model specifies. In a particular problem called part removal, which is similar to the motion planning application discussed above, one possible method of dealing with this uncertainty in size is to determine tight bounds on the physical sizes that will actually fit into the assembly without slipping out or causing unwanted motion. By first computing the upper bound on the scale of the part that will fit to the configuration in the assembly, we can then find the smallest scale for which the assembly traps it in place by specifying an escape path through the assembly and computing the largest valid part scale for which that path is valid.

\subsection{Feature detection and shape decomposition}

Minkowski sum involving a dynamically-scaling disc or sphere has strong connection to the medial axis (MA), an important shape descriptor. Follow the notation used in $\alpha$-shapes by Edelsbrunner et al. [12], we call a disc and a sphere with diameter $\alpha$, an $\alpha$-disc and $\alpha$-sphere.

Lu et al. [25] proposed a method to create shape decompositions by detecting the intersections of convolution between a polygon and a sequence of $\alpha$-discs. They show that the vertices in the arrangement of convolution are closely related to well-known features, such as bridges and pock- ets, related to the concavity of polygons. More specifically, a bridge $\beta$ of a given polygon $P$ is a segment $\beta=\overline{v u}$ that lies completely in the space exterior to $P$, where $v$ and $u$ are two points on the boundary $\partial P$ of $P$. More specifically, a segment $\overline{v u}$ is a bridge of $P$ if and only if $v, u \in \partial P$ and the open set of $\overline{v u}$ is a subset of the complement of $P$, and an $\alpha$ bridge is a bridge between the tangent points of an empty $\alpha$-disc centered at an intersection $x$ of the convolution.

By varying the value of $\alpha$, these $\alpha$-bridges are born and died, thus the persistence (i.e., life span) of bridges provide an important measurement to the significance of concave features. More specifically, when $\alpha=0$, the bridges correspond to the reflex vertices and when $\alpha=\infty$, the bridges correspond to the convex hull edges of the polygon. Interestingly, these intersections parameterized by $\alpha$ also implicitly trace out the Voronoi Complex (VC) in the space exterior to $P$. Thus, the persistence analysis corresponds to measuring the length of the segments on the VC.

In [25], the convolution and its intersections are computed from scratch when $\alpha$ changes. As shown in Appendix, there is a straightforward way to modify Section 4 to handle the situation with arcs. This approach allows us to continuously update the concave features for multiple $\alpha$ values at only critical events. The speed-up for the decomposition of the polygons shown in Fig. 12 using the proposed method is 68.556 over that of repetitive re-computation of the Minkowski sums.

\section{Conclusions and future works}

Conclusions. We have demonstrated a novel method for dynamically updating the Minkowski sum when the input polygons are uniformly scaled. Our method is orders of magnitude faster than the naïve method of recomputing the Minkowski sum from scratch, and allows rapid, continuous scaling.

Our method has direct applications in motion planning, rapid prototyping and even shape decomposition. Given the frequency with which repeated objects are used at different sizes within assemblies, or are dynamically scaled during CAD to identify a correct size, our method has significant potential to improve the quality of CAD and rapid prototyping methods. In motion planning, our method provides significant improvements to the speed of computing obstacle spaces in environments with repeated objects. The dynamic scaling of the Minkowski sum has also been demonstrated to be a useful tool for persistence analysis of important structural features.

Limitations and future works. The major limitations of enumerative strategies to scaling are that they require a large commitment of pre-processing time and memory to be effective, and that they are not usable for non-uniform scaling. In two dimensions, the pre-processing and memory footprints are typically acceptable. In $3 \mathrm{D}$ however, the worst-case boundary complexity for the Minkowski sum is $O\left(m^{3} n^{3}\right)$, while the event set is of worst-case complexity $O\left(m^{4} n^{4}\right)$, which is already impractical.

The major limitation of the non-enumerative error correction is that while it enables us to perform non-uniform scaling quickly and robustly, in its current form it is only 


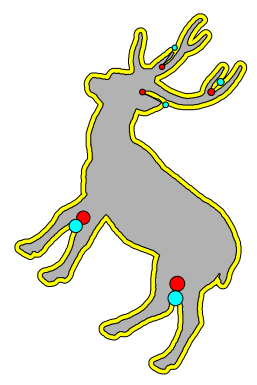

(a) $\alpha=0.05$

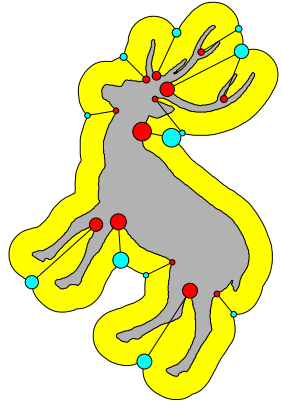

(b) $\alpha=0.2$

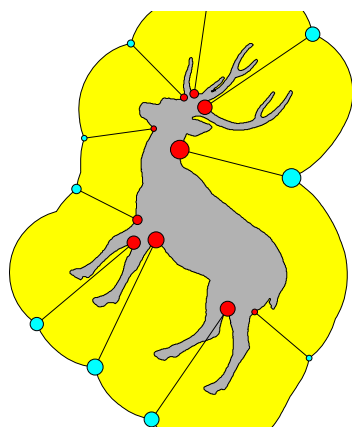

(c) $\alpha=0.4$

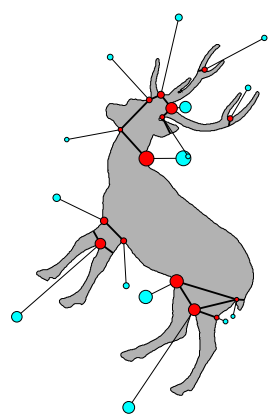

(d) $0.05 \leq \alpha \leq 1$

Fig. 12. The lighter (blue) discs on the outer boundary are convolution intersections, and the darker (red) discs on the beetle polygon are pocket minima. Corresponding pairs are connected. Several concave features disappear after $\alpha$ increases from 0.05 to 0.2 . Figure (d) shows the decompositions using persistence analysis for $0.05 \leq \alpha \leq 1$.

capable of handling convex pairs.

This work is the first step to producing generalized dynamic Minkowski sum operations under deformation of the input models. In the near future, we seek to extend our nonenumerative method to non-convex polyhedra. Such an extension would likely lead to a dynamic update method for non-convex polyhedra under rotation as well. In the long term, we will address the more complex case free-form deformation of the vertices, which introduce localized changes instead of the comparatively less complex global changes introduced by the scaling case.

\section{References}

[1] P. K. Agarwal, E. Flato, and D. Halperin. Polygon decomposition for efficient construction of Minkowski sums. In European Symposium on Algorithms, pages 20-31, 2000.

[2] F. Avnaim and J. Boissonnat. Polygon placement under translation and rotation. STACS 88, pages 322-333, 1988.

[3] H. Barki, F. Denis, and F. Dupont. Contributing vertices-based Minkowski sum computation of convex polyhedra. Comput. Aided Des., 41(7):525-538, 2009.

[4] H. Barki, F. Denis, and F. Dupont. Contributing vertices-based Minkowski sum of a non-convex polyhedron without fold and a convex polyhedron. In Shape Modeling and Applications, 2009. SMI 2009. IEEE International Conference on, pages $73-80$, june 2009.

[5] H. Barki, F. Denis, and F. Dupont. Contributing vertices-based Minkowski sum of a nonconvex-convex pair of polyhedra. $A C M$ Trans. Graph., 30:3:1-3:16, Feb. 2011.

[6] E. Behar and J.-M. Lien. A new method for mapping the configuration space obstacles of polygons. Technical Report GMU-CS-TR-2010-11, George Mason University, 2010.

[7] E. Behar and J.-M. Lien. Dynamic Minkowski sum of convex shapes. In Proc. of IEEE Int. Conf. on Robotics and Automation, Shanghai, China, May 2011.

[8] E. Behar and J.-M. Lien. Fast and robust 2d Minkowski sum using reduced convolution. In Proc. IEEE Int. Conf. Intel. Rob. Syst. (IROS), San Francisco, CA, Sep. 2011.

[9] R. Brost. Computing metric and topological properties of configuration-space obstacles. In 1989 IEEE International Conference on Robotics and Automation, 1989. Proceedings., pages 170-176, 1989.

[10] B. R. Donald. A search algorithm for motion planning with six degrees of freedom. Art. Intell., 31(3):295-353, 1987.

[11] H. Edelsbrunner. Lines in space: a collection of results. In ??, volume 6 of DIMACS Series Discrete. Math. Theoret. Computer Sci., pages 77-93. 1991.
[12] H. Edelsbrunner, D. G. Kirkpatrick, and R. Seidel. On the shape of a set of points in the plane. IEEE Trans. Inform. Theory, IT-29:551-559, 1983.

[13] E. Flato. Robuts and efficient construction of planar Minkowski sums. M.Sc. thesis, Dept. Comput. Sci., Tel-Aviv Univ., Isael, 2000.

[14] E. Fogel and D. Halperin. Exact and efficient construction of Minkowski sums of convex polyhedra with applications. In Proc. 8th Wrkshp. Alg. Eng. Exper. (Alenex'06), pages 3-15, 2006.

[15] P. K. Ghosh. A unified computational framework for Minkowski operations. Computers and Graphics, 17(4):357-378, 1993.

[16] L. J. Guibas, L. Ramshaw, and J. Stolfi. A kinetic framework for computational geometry. In Proc. 24th Annu. IEEE Sympos. Found. Comput. Sci., pages 100-111, 1983.

[17] L. J. Guibas and R. Seidel. Computing convolutions by reciprocal search. Discrete Comput. Geom., 2:175-193, 1987.

[18] P. Hachenberger. Exact Minkowksi sums of polyhedra and exact and efficient decomposition of polyedra in convex pieces. In Proc. 15th Annual European Symposium on Algorithms (ESA), pages 669-680, 2007.

[19] A. Kaul and J. Rossignac. Solid-interpolating deformations: construction and animation of PIPs. In Proc. Eurographics '91, pages 493-505, 1991.

[20] L. Kavraki. Computation of configuration-space obstacles using the fast fourier transform. IEEE Trans. Robot. \& A Autom, 11:255261, 1995.

[21] W. Li and S. McMains. A gpu-based voxelization approach to 3d Minkowski sum computation. In SPM '10: Proceedings of the 14th ACM Symposium on Solid and Physical Modeling, pages 31-40, New York, NY, USA, 2010. ACM.

[22] J.-M. Lien. Minkowski sums of rotating convex polyhedra. In Proc. 24th Annual ACM Symp. Computat. Geom. (SoCG), June 2008. Video Abstract.

[23] J.-M. Lien. A simple method for computing Minkowski sum boundary in 3d using collision detection. In The Eighth International Workshop on the Algorithmic Foundations of Robotics (WAFR), Guanajuato, Mexico, Dec 2008.

[24] T. Lozano-Pérez. Spatial planning: A configuration space approach. IEEE Trans. Comput., C-32:108-120, 1983.

[25] Y. Lu, J.-M. Lien, M. Ghosh, and N. M. Amato. $\alpha$-decomposition of polygons. Computer $\&$ Graphics, SMI 12 special issue, 2012.

$[26]$ N. Mayer, E. Fogel, and D. Halperin. Fast and robust retrieval of Minkowski sums of rotating polytopes in 3-space. In Proc. Symposium of Solid and Physical Modeling (SPM), 2010.

[27] G. D. Ramkumar. An algorithm to compute the minkowski sum outer-face of two simple polygons. In Polygons, Proc. ACM Symposium on Computational Geometry, pages 234-241. Press, 1996.

[28] E. Sacks and C. Bajaj. Sliced configuration spaces for curved planar bodies. The International Journal of Robotics Research, 17(6):639, 1998.

[29] G. Varadhan and D. Manocha. Accurate Minkowski sum approximation of polyhedral models. Graph. Models, 68(4):343$355,2006$. 
[30] R. Wein. Exact and efficient construction of planar Minkowski sums using the convolution method. In Proc. 14th Annual European Symposium on Algorithms, pages 829-840, 2006.

[31] D. Zhu and J. Latombe. New heuristic algorithms for efficient hierarchical path planning. IEEE Transactions on Robotics and Automation, 7(1):9-20, 1991.

\section{Appendix: Dynamic scaling of a disc}

We seek to update the convolution of $P$ and $Q$ when $P$ is a polygon and $Q$ is a disc, as $Q$ is scaled to some factor $s>0$. The convolution $P \otimes Q$ is produced when $s=1$, and $P \otimes s Q$ is the convolution at scale $s$. The disc $s Q$ has the same center $c$ as $\operatorname{disc} Q$, but radius of $s r$.

There are three critical events that we seek to locate in order to produce an event map over $s$, the intersections of arcs with other arcs; the intersections between line segments and other line segments; and the intersections of line segments with arcs.

Intersections of line segments with line segments Consider two line segments, $l_{1}=\left(p_{1}, p_{2}\right), l_{2}=\left(p_{3}, p_{4}\right)$. We can parameterize these as $l_{1}(t)=p_{1}+t\left(p_{2}-p_{1}\right), l_{2}(k)=$ $p_{3}+k\left(p_{4}-p_{3}\right)$. We can easily compute the intersection of these two line segments. Since line segments are always contributed by $P$, which does not scale, the update of these segments over scaling is simple to compute. Let $c$ be the center of the disc with which $l$ is convolved, and $\perp l$ be the unit vector in the direction from the center $c$, perpendicular to line segment $l$. Then, when $Q$ scales by a factor of $s$, updated reference points are computed as $p(s)=$ $p+(s-1) r(\perp l)$, and the new line segments are computed as $l_{1}(t)_{s}=p_{1}(s)+t\left(p_{2}-p_{1}\right), l_{2}(k)_{s}=p_{3}(s)+k\left(p_{4}-p_{3}\right)$.

As in Section 4, we want to compute the critical events. Because the line segments do not grow as $Q$ scales, we can express the intersection point of the lines on which they lay in terms of $s$. Because these line segments do not grow, they must have exactly two events, both of which must occur at an endpoint of at least one of the line segments, or else the line segments do not intersect at all.

Therefore, having the intersection point expressed in terms of $s$, we can proceed to set the formula equal to $p_{1}(s), p_{2}(s), p_{3}(s)$, and $p_{4}(s)$ each in turn. We can then substitute into the line equations to solve for $t$ and $k$, rejecting solutions for which $0<t$ or $t>1$, or similarly for $k$, as these intersections are not on both line segments.

These critical events allow us to find out at what scale values intersections appear and disappear. This saves us the trouble of having to check every pair of line segments between updates.

Intersections of arcs with arcs First consider the problem of two intersecting discs, $C_{1}$ and $C_{2}$ with corresponding centers $c_{1}$ and $c_{2}$, which share a radius $r$. An event occurs when $C_{1}$ and $C_{2}$ lay tangent to each other. The tangency point is the midpoint of the line segment $l=\left(c_{1}, c_{2}\right)$. Because the centers of the discs do not translate, this midpoint stays constant regardless of scale. Further, the intersections between the two discs will always lay on the line perpendicular to $\left(c_{1}, c_{2}\right)$ which passes through its midpoint.

Thus, the intersections simply move in the direction $\pm \perp$ $\left(c_{1}, c_{2}\right)$, regardless of the orientation chosen for the perpendicular. Given the unit vector, we can find the magnitude of the motion by fixing $c_{1}$, and considering a disc of radius $r$ at $\left\langle x_{c_{1}}+2 r, y_{c_{1}}\right\rangle$. Then the tangent line is the verticle line $x=x_{c_{1}}+r$, and it intersects with the discs at $\left(x-x_{c_{1}}\right)^{2}+\left(y-y_{c_{1}}\right)^{2}=\left(x_{c_{1}}+r-x_{c_{1}}\right)^{2}+\left(y-y_{c_{1}}\right)^{2}=$ $r^{2}+\left(y-y_{c_{1}}\right)^{2}=(s r)^{2}$ as the discs scale. (Remember, the $x$ coordinate stays fixed.)

As a result, the magnitude of the motion of the intersection is going to be determined as $y=\sqrt{\left(s^{2}-1\right)} r+y_{c_{1}}$. Notice that if we shrink the discs, $y$ is not real-valued, which makes sense since there is no intersection between the discs for $s<1$. We note that if the tangency occurs at some general scale $s_{0}$, we get $y=\sqrt{\left(s^{2}-s_{0}^{2}\right)} r+y_{c_{1}}$. If we let this magnitude be $M(s)$, then the update function for the intersection point is $I(s)=M(s)\left( \pm \perp\left(c_{1}, c_{2}\right)\right)$.

Arcs are not complete discs, just as line segments are not complete lines, and we can solve for critical events that do not occur at the tangency points by similarly assigning $I(s)$ to the end-points of the arc, and solving for $s$. In the case of arcs, there may be 0,1 or 2 solutions for this system.

Intersection of arcs with line segments We start with a disc and a line, instead of an arc and a line segment; the critical events for a line and a disc occur when the line lays tangent to the disc. We can compute the tangency point by computing the line intersection of the line along $\perp l$ through $c$, and $l$ itself, in terms of $s$. The points on the disc's perimeter that this line passes through will be $c \pm s r(\perp l)$. We can then compute the critical events for the line and the disc by setting these expressions equal to each other and solving for $s$, which yields $s_{0}$.

To compute the intersection points, we compute the magnitude of the change along the intersection segment $l$. Consider the tangent line $x_{0}=x_{c}-s_{0} r$. As $s$ varies from $s_{0}$, we wish to find the $y$ coordinate of the intersection between this line and the disc.

As we pass the line through the disc, the intersection height is again calculable using the disc's equation, $(x-$ $\left.x_{c}\right)^{2}+\left(y-y_{c}\right)^{2}=(s r)^{2}$, using the intersection of a vertical line with the perimeter of the disc as above. Consequently, the magnitude of the change in the intersection point is easy to update. Combined with $\perp l$, retrieving the associated displacement vector is trivial.

Finding the event points is again a simple matter of finding all real-valued solutions $s$ for the end-points of the line segment, and for the end-points of the arc. With the data structure computed, we can use it to update the convolution as in Section 4. 